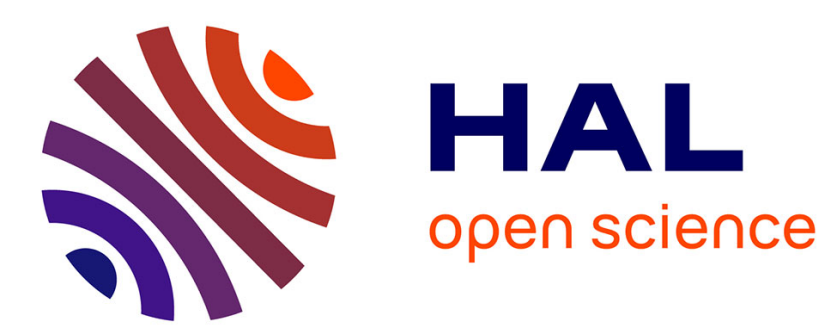

\title{
Self-similar phase space in spin glasses
}

Serge Galam, Alain Mauger

\section{- To cite this version:}

Serge Galam, Alain Mauger. Self-similar phase space in spin glasses. Journal de Physique I, 1994, 4 (3), pp.467-473. 10.1051/jp1:1994111 . jpa-00246921

\section{HAL Id: jpa-00246921 https://hal.science/jpa-00246921}

Submitted on 1 Jan 1994

HAL is a multi-disciplinary open access archive for the deposit and dissemination of scientific research documents, whether they are published or not. The documents may come from teaching and research institutions in France or abroad, or from public or private research centers.
L'archive ouverte pluridisciplinaire HAL, est destinée au dépôt et à la diffusion de documents scientifiques de niveau recherche, publiés ou non, émanant des établissements d'enseignement et de recherche français ou étrangers, des laboratoires publics ou privés. 
Classification

Physics Abstracts

$75.10 \mathrm{~N}-75.40 \mathrm{G}$

\title{
Self-similar phase space in spin glasses
}

\author{
Serge Galam and Alain Mauger \\ Groupe de Physıque des Solides, Tour 23, 2 place Jussieu, 75251 Paris Cedex 05, France
}

(Received 19 August 1993, revised 4 November 1993, accepted 17 November 1993)

\begin{abstract}
Postulating homogeneity for the relaxation function, we show that the standard dynamic scaling hypothesis is recovered and that effective relaxation times must decrease upon increasing Kadanoff's blocks sizes. In the case of spin glasses our results are naturally obtained within a diffusion process in the phase space among elgenstates avallable to the spin system. The model suggests that the critical slowing-down in spin glasses does not result from hierarchically constrained dynamics.
\end{abstract}

\section{Introduction.}

For many decades, freezing phenomena have been a fascinating puzzle in condensed matter. While a lot of experimental, numerical and theoretical efforts were devoted to its study, relaxation processes in glasses are still lacking a comprehensive explanation. If spin glasses, from the static point of view, are better understood than their structural glass counterparts, dynamics for both classes of systems are resisting analysis. At best, empirical laws are used to describe experimental data. On this basis even rather simple models may be valuable to shed light on glassy dynamics.

Glassy relaxations can often be fitted in term of a Kohlrausch law, i.e., a stretched exponential behavior. First used to fit relaxation processes in glasses and polymers [1,2], stretched exponentials have also been used to describe relaxations of the magnetization $M(t)$ in spin glasses [3] giving

$$
M(t)=M_{0} \exp \left[-\left(\frac{t}{\tau}\right)^{\beta_{\mathbf{e}}}\right] .
$$

This law being purely phenomenologic, various attempts have been made to give some physical basis to equation (1) [4-6]. In particular hierarchically constrained dynamics have been invoked [7]. The basic idea is to consider a discrete series of levels $n=0,1,2 \ldots$ with degrees of freedom at level $n+1$ represented by $N_{n+1}$ Ising spins $S_{\imath}$ assumed to be locked, except when $\mu_{n}$ spins

( ${ }^{*}$ Laboratoire associé au C.N.R.S. (U.A. $\mathrm{n}^{\circ} 17$ ) et aux Universités Parıs VII et Parıs VI. 
at level $n$ attain one particular state of their $2^{\mu_{n}}$ possible ones. It follows that the times needed to relax these $N_{n+1}$ spins and $N_{n}$ spins at levels $n+1$ and $n$ respectively are related by [7],

$$
t_{n+1}=2^{\mu_{n}} t_{n}
$$

The Kohlrausch law is then recovered, provided relations $\mu_{n}=\mu_{0} n^{-p}$ and $N_{n+1}=\frac{N_{n}}{\lambda}$ are assumed [7]. However, they are truly ad-hoc relations arbitrarily chosen in order to recover the stretched exponential behavior. The model has thus only shifted the initial problem of understanding equation (1) to that of understanding the recursion relations on $\mu_{n}$ and $N_{n+1}$. Moreover the loose definition of "levels" hinders insight in the physics of spin glass freezing.

In this paper a time-extended renormalization group approach is presented to account for a scaling of block relaxations. It is shown that the dynamic scaling hypothesis amounts to the assumption of homogeneity for the relaxation time function. In the case of spin glasses, this assumption leads naturally to relate relaxation to a diffusion phenomenon in phase space [6]. No hierarchical feature is used at contrast with previous models based on a hierarchical dynamics hypothesis [8].

It is also argued that our non-hierarchical model produces, for spin glasses, a value of the dynamic exponent consistent with its experimental counterparts. It is already known from the diffusion model [6], that the same holds true for the stretched exponential parameter $\beta_{e}$. On the opposite it is worth stressing that hierarchical models fail to predict any value for $z$ and g1ve a value $\beta_{\mathrm{e}}$ which disagrees with the experimental estimate near the spin glass freezing temperature.

The paper is organized as follows. A time-extended renormalization group treatment is reported in section 2. Section 3 is devoted to the derivation of the dynamic exponent within the diffusion model where our hypothesis is fulfilled. Results are then compared to experimental data. Hierarchical versus non hierarchical dynamics are discussed in section 4. Last part contains concluding remarks.

\section{Dynamics within renormalization group.}

We start with a $d$-dimensional lattice with lattice spacing $u_{0}=a$. At each site is associated a spin. To perform a b-step real space renormalization group or Kadanoff transformation, original degrees of freedom are first grouped into blocks of $b^{d}$ spins. Then, each block of $b^{d}$ spins is replaced by only one spin, the value of which is calculated using some majority rule. So doing, a new effective lattice is obtained with a new lattice spacing $u_{1}=b u_{0}=b a$. In the process the effective correlation length, which is measured in units of the lattice spacing, has been divided by the factor b, with $\xi_{1}=b^{-1} \xi_{0}$. Repeating the transformation again and again, effective levels $n$ and $n+1$ are related by

$$
u_{n+1}=b u_{n}=b^{n+1} u_{0} \text {. }
$$

It is noteworthy that actual physical correlation length $\xi$ is unchanged under the renormalization process, so that

$$
\xi=\xi_{n+1} u_{n+1}=\xi_{0} u_{0}
$$

equations (3) and (4) imply

$$
\xi_{n+1}=b^{-1} \xi_{n}=b^{-(n+1)} \xi_{0} .
$$

In this scheme, the $\left\{\xi_{n}\right\}$ are dimensionless quantities which measure the effective number of degrees of freedom per correlation length. 
To include the dynamics in the renormalization group or Kadanoff transformation we introduce an effective unit of time by analogy with the effective lattice spacing. The physical relaxation time $\tau$ can then be expressed as

$$
\tau=\tau_{0} t_{0}
$$

where $t_{0}$ is the time unit associated with the initial spin and $u_{0}$ lattice spacing. $\tau_{0}$ is the corresponding dimensionless relaxation time. At stage $n$, i.e., in effective lattice $u_{n}$, there exists a unit time $t_{n}$ and a dimensionless relaxation time $\tau_{n}$ satisfying the time analog of equation (4), $\tau=\tau_{n} t_{n}$. At this stage the question is to express $\tau_{n+1}$ as function of $\tau_{n}$, or indeed $t_{n+1}$ as function of $t_{n}$.

To derive it we generalise the static scaling hypothesis by postulating the homogeneity property of the singular part of the free energy to the relaxation function. This assumption implies for the recursion relations the following form,

$$
t_{n+1}=b^{x} t_{n}=b^{(n+1) z} t_{0}
$$

and,

$$
\tau_{n+1}=b^{-z} \tau_{n}=b^{-(n+1) z} \tau_{0}
$$

with $z$ the dynamic exponent. These equations are the time counterparts of equations (3) and (5). Equation (7) has been already derived in the dynamic renormalization group theory, and successfully applied to the analysis of Monte Carlo configurations in various systems [9]. It is, however, $\tau_{n}$ which is the relevant time in a renormalization group procedure. Although, intuitively, one would have expected larger relaxation times for larger blocks of spins, it must be stressed that in the renormalization process, larger blocks means indeed less and less degrees of freedom per correlation length after equation (5), making smaller relaxation times logical, hence $y>0$.

Eliminating $b$ and $n$ from equations (5) and (8) gives,

$$
\frac{\tau_{n+1}}{\tau_{0}}=\left(\frac{\xi_{n+1}}{\xi_{0}}\right)^{z} .
$$

This is the classical form for the conventional dynamic scaling hypothesis. Note we have deduced equation (9) from equations (5) and (8). Instead, one might have started from equations (5) and (9) to derive equation (8). Therefore, equation (8) which results from our hypothesis of homogeneity of the relaxation function, is equivalent to the standard dynamic scaling hypothesis which postulates equation (9). Although this scaling hypothesis has been subject to debate in the recent past, there is now an overall agreement (and an experimental evidence) that it is indeed relevant to spin glasses [10]. In any case, if equation (9) holds, then equation (8) is the only possible choice for time rescaling in the building of blocks in a renormalization scheme. In others words, relaxation times must decrease with increasing block sizes.

On another hand, we have argued [11] that it is enough to postulate homogeneity for the singular part of the free energy to get the static scaling hypothesis under the form

$$
\frac{\xi_{0}}{\xi_{n}} \propto \tilde{t}^{-\nu}
$$

with $\nu$ given by the Josephson hyperscaling relation [12] $d \nu=2-\alpha$ in terms of the specific heat exponent $\alpha$. 


\section{About relaxation as diffusion.}

Relaxation in a $N$-spin system is related to the landscape of available states in the phase space. Our hypothesis of homogeneity of the relaxation function implies that this landscape satisfies self-similarity. To achieve this property we must have either criticality or a fractal geometrical structure in the phase space. This last property appears indeed to be realized in the diffusion model [6]. This model has been built for Ising spins, but we will not distinguish between Ising and Heisenberg systems, as there are now arguments that they both have the same critical behavior and belong to the same class of universality [13]. In the diffusion model where the system relaxes by single spin-flips, diffusion occurs via diffusion from vortex to nearest-neighbor vortex in the $N$-dimensional hypercube of the spin phase-space [14]. At high temperatures, i.e. in a paramagnetic configuration, available eigenstates cover densely the hypercube. In the case of long-range ordering like in ferromagnets, upon cooling the system can pick up the ground state which is only two-fold degenerate due to up-down symmetry. At contrast, in spin glass systems, the ground state is non trivial. Frustration generates a thermodynamically significant number of locally metastable states $(\alpha \exp \alpha N)$ [15]. The increase of associated energy barreers upon cooling makes some minima not reachable, making the $N$-dimensional hypercube effectively diluted. As a result, at some temperature $T_{\mathbf{r}}$, a crossover occurs from the dense structure for available states to a ramified structure which still percolates. Upon further cooling, the density of available states on the hypercube may reach at some finite temperature $T_{\mathrm{c}}$, the percolation threshold [6]. Such a $T_{\mathrm{c}}$ exists only for real space dimensions higher than the lower critical dimension of these sytems, which is believed to be 2 [16]. The critical slowing down is thus observed in the range $T_{\mathrm{c}}<T<T_{\mathrm{r}}$. Indeed, the ramified structure above mentioned in the spin-phase space has a fractal dimension [6], and thus the relaxation function is homogeneous. To check the validity of our model we are now in position to determine the values of critical exponents within a diffusive relaxation mechanism and then, to compare the results to experimental values.

3.1 ThE DIFFUSION MODEL. - Estimates of critical exponents can be obtained from prior works on other fractal lattices where a finite $T_{c}$ exists. For such systems, Rammal argued that the standard dynamic scaling hypothesis (Eq. (5)) holds true, with a constant value for $z$ [16]. For $\bar{d}=1+\epsilon$, with $\epsilon$ smaller than 1 , dynamics will be dominated by the 1 -d diffusion of domain walls, which leads to $z=2+\epsilon[17]$, hence $z=\bar{d}+1$. In our problem, the phase space of dimension $N$ goes to infinity in the thermodynamic limit. Aharony et al. [18] determined the associated percolation threshold fractal dimension $\vec{d}=4$, which gives

$$
z=5 \text {. }
$$

Note, however, that the value $z=5$ needs a numerical check, since its derivation lies on the Rammal hypothesis that the relation $z=\bar{d}+1$. derived for $\epsilon<1$ can be extended to much larger values of $\epsilon$, in particular $\epsilon=3$ in the present case.

3.2 ExPERIMENTS. - Measurements of the dynamic properties fail to give access to $z$ alone, because the critical slowing-down is sensitive to the product $z \nu$ only. This is apparent, for instance, after equations $(9,10)$ from which we infer $\frac{\tau_{0}}{\tau_{n}} \propto \tilde{t}^{-z \nu}$ The experimental value of $z \nu$ for spin glasses, deduced from dynamic scaling analysis is [10]

$$
z \nu=9.5
$$


Extraction of $z$ thus requires the determination of the static exponent $\nu$. For this purpose, we consider the static scaling law for the non linear magnetization, traditionally expressed as [19],

$$
M-\chi_{0} H=\frac{H}{T} \tilde{t}^{-\beta} \mathcal{F}\left[\frac{H^{2}}{T} \tilde{t}^{-(\gamma+\beta)}\right],
$$

with $\beta$ the critical exponent for the order parameter, and $\phi=\gamma+\beta$ the crossover exponent. The function $\mathcal{F}$ has a universal shape. Experimental values of $\gamma$ and $\beta$ deduced from the analysis of the magnetization data according to equation (13) in various spin glasses are [13, $20,21]$

$$
\gamma=3.3 \pm 0.3 \text { and } \beta=0.8 \pm 0.2 .
$$

When combined with the Josephson hyperscaling relation, the Essam-Fisher hyperscaling relation [22], $\alpha+\gamma+2 \beta=2$, derived from the static scaling hypothesis can be written as, $\gamma+2 \beta=d \nu$. For $d=3$, we thus get $\nu=1.7 \pm 0.2$, which, combined with equation (12) leads to

$$
z=5.58 \pm 0.9 \text {. }
$$

The consistency of this result with equation (11) gives support to the diffusion model for relaxation. The determination of $\beta_{\mathrm{e}}$ provides a second support to this model. Campbell [6] argued that, within the diffusion model, relaxation is a stretched exponential in the whole range $T_{\mathrm{c}}<T<T_{\mathrm{r}}$. Upon cooling, $\beta_{\mathrm{e}}$ decreases from 1 at $T_{\mathrm{r}}$, down to be $\beta_{\mathrm{e}}=2 /(2+\bar{d})$ at $T_{\mathrm{c}}$. Having $\bar{d}=4$ at the percolation threshold in the phase space, i e. at $T_{\mathrm{c}}$, the diffusion model thus predicts [6],

$$
\beta_{\mathrm{e}}=\frac{1}{3} \text { at } T=T_{\mathrm{c}} .
$$

This value is not only in agreement with simulation results [23], but also with experimental determinations of both $\beta_{\mathrm{e}}=0.34 \pm 0.05$ in $\mathrm{Eu}_{0.4} \mathrm{Sr}_{0.6} \mathrm{~S}$ just above $T_{\mathrm{c}}$ [24], and $\beta_{\mathrm{e}}=\frac{1}{3}$ around $T_{\mathrm{c}}$ in $\mathrm{CdIn}_{0.3} \mathrm{Cr}_{1.7} \mathrm{~S}_{4}[25]$.

\section{Hierarchical versus non hierarchical dynamics.}

The non hierarchical model displayed in section 2 relies on the time recursion relation in equation (8). Indeed we have shown that equations (8) and (9) which are equivalent, result from the hypothesis of homogeneity of the relaxation time function. Since the diffusion model is based on a fractal lattice at $T_{\mathrm{c}}$, it automatically satisfies the property of self-similarity, which in turn implies homogeneity. Therefore, the diffusion model realizes our model of section 2. Then, only the static scaling hypothesss needs to be added to the diffusion model to give a full description of the spin glass freezing. Comparing with experiments, Campbell has already found that the model accounts for the stretched exponential relaxation with $\beta_{e}=1 / 3$ in agreement with the exper imental value. In addition, we have found that, if we admit the Rammal hypothesis, the dynamic exponent is $z=5$. This value for the dynamic exponent is also consistent with experiments.

In this model below $T_{c}$, there exist arguments [6] that the allowed phase space is even not fractal. This explains that $\beta_{\mathrm{e}}$ rapidly differs from $1 / 3$. The same should hold true for $z$. Indeed, if one attempts to derive a value of $z$ from the dynamic scaling analysis, a very sharp increase of $z \nu$ is found upon cooling below $T_{\mathrm{c}}$ [26]. However, the scaling laws used to derive $z \nu$ lies on the linear response approximation which is no longer valid at such temperatures. Therefore, it is not clear whether this sharp variation of $z \nu$ is consequent to a deviation with respect to the linear response approxımation [26], or it is due to the loss of fractal properties. Deviations 
with respect to $z \nu=10$ are also observed upon heating above $T_{\mathrm{c}}$. However these deviations are driven by relaxation of the uncorrelated magnetic clusters which mask the critical slowing-down [27].

In parallel, hierarchical dynamics have been invoked recently to explain the non exponential relaxation in spin glasses [8]. In this work, Castaing and Souletie also start from a dynamic renormalization group theory, but they substitute the time recursion relation [9] displayed in equation (7) by the relation [7] displayed in equation (2). The block procedure of renormallzation group replaces any $n$-cluster of spins standing for Kadanoff's block by one superspin, whose sign is determined by the majority of original spin orientations in the original n-block. Then each superspin flips, on the average, after a time which is the unit time $t_{n}$ (unfortunately called $\tau_{n}$ in Ref. [8]). The hierarchy principle in equation (2) means that, at stage $n+1$, the renormalized spin of the $\mathrm{n}+1$ - cluster can change its state only if $\mu_{n}$ of the Ising superspins at stage $n$ are in a given state. Of course, equation (2) can still be put under the form of equation (7), with $z=\mu \frac{\ln 2}{\ln b}$, and $\mu$ the average value of the $\mu_{n}$ over $n$. With such notations, the non hierarchical model in section 2 corresponds to the case where $\mu \propto \ln b$ in order to insure that $z$ is independent of $b$, as it should be in a renormalization procedure. However, this relation between $\mu$ and $b$ cannot be justified within the hierarchical model, which is built on independent $\mu$ and $b$, and indeed, the novelty introduced by the hierarchy is that the critical exponent $z$ now depends on $b$. One consequence is that the critical slowing-down of spin glasses should not be universal, i.e. each spin glass material should have a particular critical exponent $z$, depending on the particular value of the length scale $b$ relevant to the problem, according to the concentration of magnetic ions in the matrix, on the magnetic interactions, and on the geometrical topology of the lattice. On an experimental point of view, however, recent experiments in insulating spin glasses $\mathrm{Hg}_{1-x} \mathrm{Mn}_{x} \mathrm{Te}, \mathrm{Cd}_{1-x} \mathrm{Mn}_{x}$ Te show the critical dynamics are the same, whether $x=0.3$ or $x=0.4$, whether the cation is $\mathrm{Hg}$ or $\mathrm{Cd}$ [28]. The critical properties are also the same in these Heisenberg materials with short range antiferromagnetic interactions, and in $\mathrm{Eu}_{1-x} \mathrm{Sr}_{x} \mathrm{~S}$ with competing ferro and antiferromagnetic interactions between nearest and next nearest neighbors, respectively. The critical properties are also the same in these systems which crystallize in the fcc lattice, and in aluminoslicates which are amorphous materials. There is thus experimental evidence that the spin glasses form a broad class of universality, which pleads in favor of the non-hierarchical model. Another argument in favor of this model is that it yields a numerical estimate of the coefficient $\beta_{\mathrm{e}}$ entering equation (1) equal to $1 / 3$, in agreement with experiments. At contrast, within the hierarchical model, $\beta_{\mathrm{e}}^{-2} \propto \ln \left(\frac{\xi}{a}\right)$ after reference [8], yielding $\beta_{\mathrm{e}}=0$ at $T_{\mathrm{c}}$.

\section{Conclusion.}

In conclusion, we have argued that the dynamic scaling hypothesis results from homogeneity of the relaxation time function. Scaling relation among relaxation times of Kadanoff's blocks in real space is obtained. The characteristic times decrease with increasing the block size. We have shown that this relation is achieved within the diffusion model according to which the system relaxes by single spin flips among available states constituting a percolating (but not dense) ramified structure in phase space. In particular the relaxation in this regime $\left(T \geq T_{\mathrm{c}}\right)$ is not the result of hierarchically constraned dynamics. This, of course, does not precludes the possibility that a hierarchy dominates the relaxation in the ordered phase. The resulting critical slowing down in spin glasses is consistent with the experımental results available on three-dimensional spin glasses. 


\section{Acknowledgments.}

The authors would like to thank D. Stauffer for stimulating discussion.

\section{References}

[1] Kohlrausch R., Ann. Phys. (Leipzig) 12 (1847) 393.

[2] Struick L. C. E., Physıcal Aging in Amorphous polymers and other materials (Elseiver Scientific Publishing Company, 1978) p. 1.

[3] Mezei F. and Murani A.P., J. Magn. Mater. 14 (1979) 211.

[4] Ngai K. L., Comments Solid Stat. Phys. 9 (1979) 127 and 9 (1980) 141.

[5] Cohen M. M. and Grest G.S., Phys. Rev. B24 (1981) 4091.

[6] Campbell I. A., J. Phys. Lett. 46 (1985) 1.1159.

[7] Palmer R. G., Stein D. L., Abrahams E. and Anderson P. W., Phys. Rev. Lett. 53 (1984) 958.

[8] Castaing B. and Souletie J., J. Phys. France 1 (1991) 403.

[9] Jan N., Moseley L. L. and Stauffer D., J. Stat. Phys. 33 (1983) 1.

[10] Bertrand D., Mauger A., Ferré J. and Beauvillain P., Phys. Rev. B45 (1992) 507.

[11] Galam S. and Mauger A., to be published

[12] Ma S.- K., Modern Theory of Critical Phenomena (Benjamin Company, Realding, Mass., 1976).

[13] Mauger A., Villain J., Zhou Y., Rigaux C., Bontemps N. and Ferré J., Phys. Rev. B41 (1990) 4587.

[14] See for instance, Ogielski A. T., Phys. Rev. B32 (1986) 7384.

[15] Bray A. J. and Moore M. A., J. Phys. C14 (1982) 2629.

[16] Southern B. W. and Young A. P., J. Phys. C10 (1977) 2179.

[17] Rammal R., J. Phys. 46 (1985) 1837.

[18] Aharony A., Gefen Y. and Kapitulnick A., J. Phys. A17 (1984) L197.

[19] Suzuki M., Prog. Theor. Phys. 58 (1977) 1151.

[20] Omarı R., Prejean J. J. and Souletie J., J. Phys. France 44 (1983) 1069.

[21] Mauger A., Ferré J. and Bauvllain P., Phys. Rev. B40 (1989) 862.

[22] See, for example, Stanley H. E., Introduction to Phase Transitions and Critical Phenomena, W. Marshall and D. H. wilkinson Eds. (Oxford University Press, New York and Oxford, 1971) p 185.

[23] Campbell I. A., Flesselles J. M., Julien R. and Botet R., J. Phys. C : Solid Stat. Phys. 20 (1987) L47.

[24] Bontemps N. and Orbach R., Phys. Rev. B37 (1988) 4708.

[25] Refrigier P., Vincent E., Ocio M. and Hamman J., Jpn J. Appl. Phys. suppl. 26783 (1987) 3.

[26] Bontemps N., Ferré J. and Mauger A., J. Phys. Colloq. France 50 (1989) 1063.

[27] Ayadi L., Ferré J., Mauger A. and Triboulet D., Phys. Rev. Lett. 57 (1986) 1165.

[28] Zhou Y., Rigaux C., Mycielski A., Menant M. and Bontemps N., Phys. Rev. B40 (1989) 8111. 